\title{
Photonic Materials for Optical Communications
}

\section{Hartmut Hillmer and Roland Germann, Guest Editors}

\begin{abstract}
An overview of key materials for optical communications, including semiconductors, dielectrics, glasses, and organics, is presented in this issue of MRS Bulletin. Materials quality is in all cases crucial for advanced device and system performance. Materials properties and important problems are reviewed, and their impact on the performance of state-of-the-art optical devices is assessed and demonstrated by means of selected examples.
\end{abstract}

Keywords: dielectrics, glasses, optical communications, organic materials, photonics, semiconductors.

The 20th century can be considered the century of electronics. The invention of the field-effect transistor in 1926 and the bipolar transistor in 1947, and the development of integrated circuits since 1958, have opened up our information age. As for the 21st century, many experts believe that it will be devoted to optics and nanostructures. Thus, we probably stand at the entrance to a century of photonics and nanosystems technology. Recent advances in modern photonics have always been strongly related to advances in materials fabrication and the understanding of the physics involved. Today's application of photonic devices, components, and systems has recently spread to an enormously wide field, encompassing communications technology (datacom and telecom); illumination technology; indicator elements (i.e., control LEDs in the automotive field, measurement equipment, audio components, etc.); self-illuminating displays; projection displays such as digital micromirror technology and laser TV; opticalstorage technology; medical technology in diagnostics, health monitoring, and surgery; devices and systems for sensing and high-precision measurements, including environmental control as well as gas and liquid detection; high-precision alignment and distance control, collision-avoiding mobile systems; and finally, direct laser applications for cutting, welding, soldering, and drilling.

This issue is devoted to photonic materials for optical communications. A broad set of active and passive devices is used in today's optical communications systems. The optical part of such systems consists of light emitters and receivers, a medium for the light transmission, and devices for manipulating light in the optical domain.

Light emitters are usually lasers or LEDs made of III-V semiconductor materials. Wavelengths around $1550 \mathrm{~nm}$ have become standard for long-haul applications in optical backbone networks because of the minimum propagation loss of optical fibers in this wavelength window. Shorter-range applications in local networks, optical interconnects, optical backplanes, and even on-board and on-chip communications are using shorter wavelengths, in the range of $1310 \mathrm{~nm}, 980 \mathrm{~nm}$, $850 \mathrm{~nm}$, or below. The writing and reading of information in storage devices, such as compact disks and digital versatile disks, can be considered a special kind of shortrange optical communication that makes use of even shorter wavelengths, ranging from $850 \mathrm{~nm}$ to less than $400 \mathrm{~nm}$.

The optical receiver in optical communications systems is also dominated by semiconductor materials, such as silicon and compound semiconductors. Different types of photodiodes cover the wavelength range between $1600 \mathrm{~nm}$ and the near-UV region.

The vast majority of optical communications systems use optical fibers as the transmission medium. Optical free space communication in air is used in special cases, such as short-distance data links or short-range optical interconnects. Glass has evolved as the material of choice for optical transmission, with doped silica being the dominant material used in singlemode fibers (SMFs) for long-haul applications. Silica multimode fibers are used for shorter-range data communications, and plastic optical fibers have gained interest as an alternative for low-cost applications.

During the last few years, we have seen an increasing demand for optical devices that can be used to manipulate light in the optical domain. Optical functions such as splitting and combining of light, switching of channels in the space domain, multiplexing and demultiplexing of channels in the wavelength domain, and filtering are very important building blocks toward the construction of all-optical networks. The advantage of these fully transparent optical networks is that the signals stay in the optical domain without the need to convert them from the optical to the electrical domain and back to perform switching operations electrically. Different technologies are used to construct such passive optical devices. Fusion of silica fibers is used to build simple devices such as power splitters and combiners. Miniature optics, such as gratings, lenses, beam splitters, prisms, mirrors, and interference filters, are used to fabricate bulk optic devices with the functionality of wavelength multiplexers and demultiplexers, switches, and filters. Although these devices exhibit very good optical properties, their future potential for certain applications may be limited because of the complicated packaging and fiber-alignment schemes, their size, and limits in integration density. Planar lightwave circuits (PLCs) based on layers of glass, polymers, or other materials deposited on a planar substrate are a promising alternative, with the potential for low-cost manufacturing and high integration density by using well-established fabrication technologies from the silicon industry.

A big industry, consisting of companies with a broad portfolio of different materials technologies and smaller niche players, has been built to supply the huge variety of devices for the optical communications sector. Although the optical device indus- 
try has been hit hard economically by the bursting of the Internet bubble, we think that the whole sector will move back to steady and healthy growth after a consolidation phase.

Because of the huge variety of different materials systems (element, III-V, and II-VI semiconductors; organic conducting, semiconducting, and insulating materials; inorganic and organic glasses; $\mathrm{LiNbO}_{3}$; dielectrics, garnets, and metals) used in the optical device industry, it is impossible to cover all materials systems and relevant materials aspects in this issue of $M R S$ Bulletin. We have tried to review a broad set of materials that are used to fabricate the most important devices of optical communications systems. This comprises III-V semiconductors for lasers, LEDs, and photodetectors, organic materials for active and passive devices, silica materials for standard SMFs, dielectric multilayer systems for filter applications, and silicabased materials for PLCs (see Table I). The articles in this issue review materials properties and fabrication technologies and try to link basic materials characteristics to device performance.
We have tried to avoid overlap with aspects of the topic that have already been covered in previous issues. We have therefore left out any aspects linked to doping with rare-earth elements [see MRS Bulletin 24 (9) (1999) pp. 16-56]. Optical fibers made of microstructured silica have also been treated earlier [MRS Bulletin 26 (2001) pp. 608-646], as have vertical-cavity surface-emitting lasers (VCSELs) [MRS Bulletin 27 (2002) pp. 497-537]. Optical devices based on microelectromechanical systems (MEMS) were reviewed in MRS Bulletin 26 (2001) pp. 282-340.

In this issue, the contribution from $\mathrm{Tu}$ and $\mathrm{Yu}$ covers element semiconductors and arsenide-based, phosphide-based, and dilute nitride-based III-V semiconductors for photonic applications roughly between $600 \mathrm{~nm}$ and $2000 \mathrm{~nm}$ wavelengths. A strong emphasis is placed on advanced light sources and photodetectors. Current trends are aimed toward achieving GaAs-substrate-based VCSELs at $1.3 \mu \mathrm{m}$ and potentially also at $1.55 \mu \mathrm{m}$.

The article by Hangleiter reviews the success story and some mysteries of Group III nitride semiconductors for LED and laser applications, mainly in the wavelength range between $350 \mathrm{~nm}$ and $500 \mathrm{~nm}$, which have created an immense market. For these materials, it is important to counteract enormous dislocations produced by heteroepitaxial growth and enforce improvements in $p$-doping. Physical details, materials properties, and device potential are emphasized.

Fuhrmann and Salbeck describe the new world of organic materials for photonic applications. Materials aspects and the wide range of options for tailoring the desired optical and mechanical properties are highlighted. Organic light-emitting diodes are given special emphasis, and other applications in optical communications such as organic solid-state lasers, optical switching, and data storage are covered as well.

Guenot's article on glass fibers for optical transport gives a short overview of fibers based on different materials systems and concentrates mainly on standard SMFs made of doped silica for long-haul telecommunications application in the $1550 \mathrm{~nm}$ wavelength window. Guenot shows in detail how different intrinsic and

Table I: Material Systems Used for Optoelectronic Device Applications.

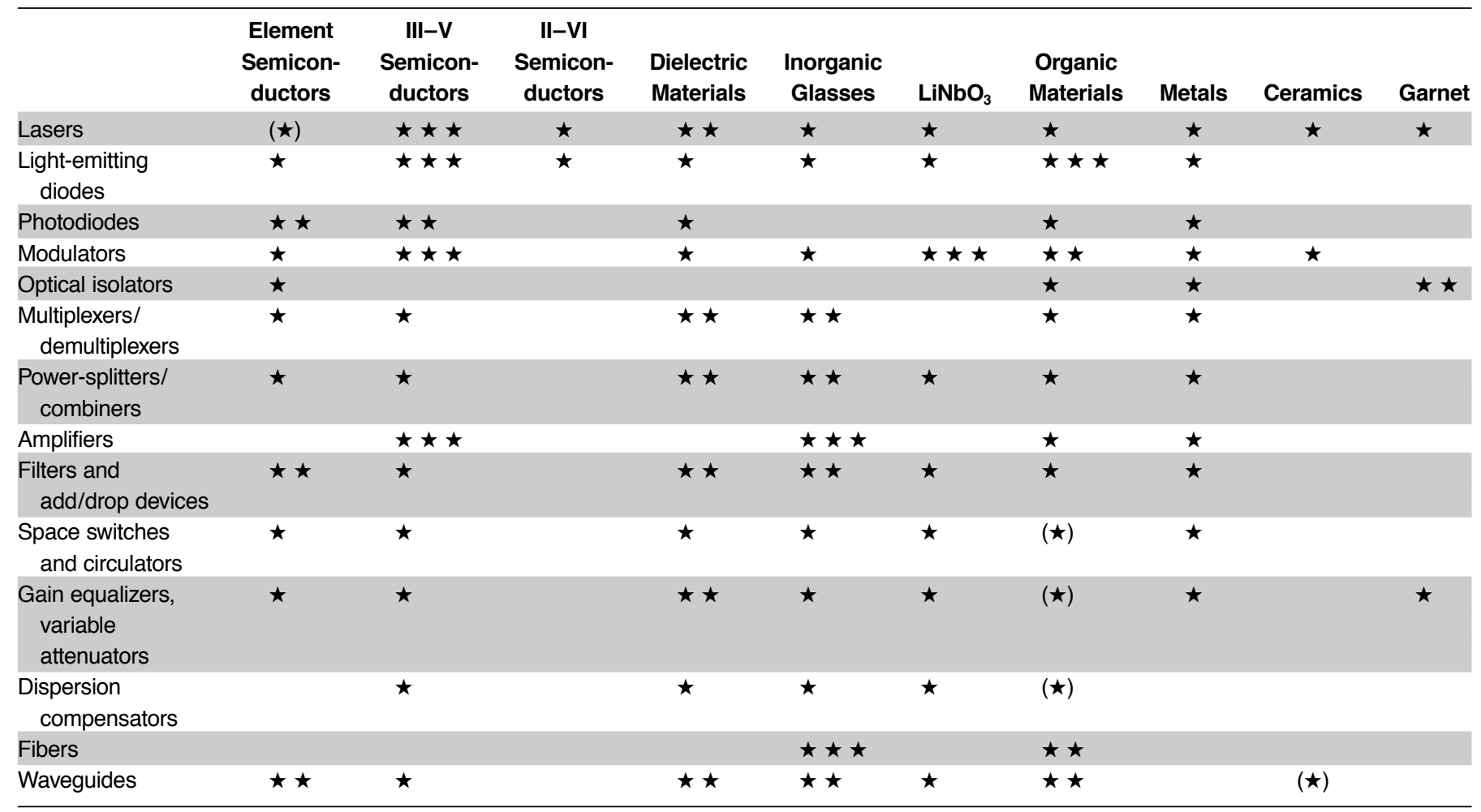

Note: The relative importance of a material system for a certain device application is indicated by the number of stars, from ( $\star$ ) $=$ less important to $\star \star \star=$ very important, and represents the authors' view of the current status. Pure electronic devices are not included. 
extrinsic loss contributions influence the fabrication of fibers with ultralow optical propagation losses.

The contribution from Hibino covers planar lightwave circuits based on doped silica glass. He describes fabrication technology and the arrayed waveguide grating multiplexer as a device example. The author also discusses ways to overcome polarization dependence, which is a key issue for planar optical devices. Special emphasis is placed on the description of a route toward higher integration densities. This is possible through increasing the doping level of the germanium-doped silica core layer, which leads to a higher refractive-index contrast between the core and cladding layers and thus to a smaller minimum bending radius of the waveguides. An alternative approach that leads to even smaller bending radii and higher integration densities is to use silicon oxynitride $(\mathrm{SiON})$ as the material for the core layer. $^{1,2}$

Sargent and $\mathrm{O}^{\prime}$ Brien describe thin-filmbased optical filter technology based on multilayer stacks of refractory oxides, which are important materials for passive optical devices and for reflectivity modification of optical surfaces in general. The authors review materials systems and the fabrication technology used in the production of thin-film-based optical filters for applications in wavelength-division multiplexing.

In nearly all cases, material qualities such as purity, homogeneity, and crystalline perfection have been found to be crucial for powerful device components and systems. Examples are extremely lowlight-absorbing optical fibers, the emission efficiencies of organic LEDs and lasers, and the high quantum efficiencies of arsenide- and phosphide-based conventional semiconductor LEDs and lasers. However, astonishing exceptions also exist, such as the poor crystalline quality of Group III nitride semiconductors, re- vealing extremely high dislocation and defect densities but nevertheless an unexpectedly high optical-light output.

On the other hand, many materials classes allow researchers and device developers tremendous freedom to combine different materials in complicated geometric constructions, thus enabling heterointerfaces in nearly arbitrary orientations in three-dimensional space by involving epitaxy, deposition, etching, and other processes.

In the past few years, there has been intense activity in the field of Group III nitride semiconductors and organic materials. Enormous impetus for full-color LED displays and high-density optical storage has been provided by the implementation of blue LEDs and blue semiconductor lasers. Another breakthrough was the development of a new generation of white LEDs and organic LEDs. Further cost reductions will take place, opening up a revolution in illumination technology in the public and private sectors. Major advances have also been made in the field of modern optical-fiber technology. Materials such as polymers, silica glass, and fluoride glass now cover a tremendous spectral range, enabling short-distance as well as long-haul fiber communications. With significant advances in ultrafast semiconductor lasers and the development of VCSELs, attractive compact ultrabright coherent light sources are available for highdata-capacity communications systems.

However, considering these successes in photonics, it is important to mention that directly generated green laser light is not commercially available. II-VI semiconductors and Group III nitrides did not succeed in opening up this spectral range without restrictions. It is worth noting that efficient green nitride LEDs are available by the help of the quantum-confined Stark effect, shifting the emission from the bluegreen to longer wavelengths. Unfortunately, this support does not hold for lasers being characterized by almost flatband conditions above threshold. Once again, this underlines the importance of understanding the relevant physics in materials science.

In optoelectronics, the band discontinuities (band lineups) and bandgaps of inorganic and organic semiconductor heterostructures play a crucial role. However, in most cases, lineup engineering and bandgap engineering are not independent from each other. This often causes serious restrictions in device design. However, interesting and attractive exceptions exist: adding $\mathrm{N}$ to GaInAs reduces the energy of the conduction-band edge, and introducing $\mathrm{Sb}$ into GaInAs energetically increases the valence-band edge. Concerning the importance of band discontinuities $^{3}$ in optimizing device performance, interesting parallels are observed in inorganic and organic semiconductor devices.

Finally, micromachining is of increasing importance in current and emerging optoelectronic systems ${ }^{4}$ and will likely bring further interesting and new materials aspects into photonics. Also, photonic crystals are a welcome addition to photonic device applications, since they bring the promise of tremendous gains in functionality. Thus, we are convinced that photonics will have an opalescent future and most probably will become one of the technological highlights of the 21st century.

\section{References}

1. B.J. Offrein, F. Horst, G.L. Bona, R. Germann, H.W.M. Salemink, and R. Beyeler, IEEE Photon. Technol. Lett. 12 (2000) p. 504.

2. R. Germann, H.W.M. Salemink, R. Beyeler, G.L. Bona, F. Horst, I. Massarek, and B.J. Offrein, J. Electrochem. Soc. 147 (2000) p. 2237.

3. H. Hillmer and S. Marcinkevicius, Appl. Phys. B 66 (1998) p. 1.

4. H.P. Herzig, H. Iawoka, N. Tien, and J. Mohr, eds., Tech. Digest Optical MEMS Conf. (Institute of Electrical and Electronics Engineers, Piscataway, NJ, 2002).

\author{
Hartmut Hillmer, Guest \\ Editor for this issue of \\ MRS Bulletin, is a pro- \\ fessor within the electri- \\ cal engineering faculty \\ and chair of Technologi- \\ cal Electronics at the \\ University of Kassel, \\ Germany. His current \\ research interests are \\ technological implemen- \\ tation, characterization, \\ and theoretical model- \\ ing of optoelectronic
}

\begin{tabular}{|c|c|}
\hline $\begin{array}{l}\text { III-V semiconductor } \\
\text { devices; spectral tuning } \\
\text { of semiconductor lasers } \\
\text { and filters; and micro- } \\
\text { systems and nano- } \\
\text { systems technologies. } \\
\text { He received a diploma } \\
\text { degree in physics in } \\
1985 \text { and his PhD de- } \\
\text { gree in physics in 1989, } \\
\text { both from the Univer- } \\
\text { sity of Stuttgart. He did } \\
\text { his habilitation thesis in } \\
1996 \text { at Darmstadt Uni- }\end{array}$ & $\begin{array}{l}\text { versity of Technology. } \\
\text { From } 1989 \text { to } 1998, \\
\text { Hillmer worked in } \\
\text { the Research Center at } \\
\text { German Telekom as } \\
\text { head of the molecular- } \\
\text { beam epitaxy facilities } \\
\text { and deputy of the } \\
\text { optoelectronics facility, } \\
\text { researching chirped } \\
\text { distributed-feedback } \\
\text { gratings by means of } \\
\text { bent waveguides, ultra- } \\
\text { fast lasers, and MBE }\end{array}$ \\
\hline
\end{tabular}

III-V semiconductor devices; spectral tuning of semiconductor lasers and filters; and microsystems and nanosystems technologies. He received a diploma degree in physics in 1985 and his PhD degree in physics in 1989, sity of Stuttgart. He did 1996 at Darmstadt Uni- versity of Technology. growth of AlGaInAs strain-compensated quantum well laser structures. In 1991, he was a visiting researcher at NTT Optoelectronics Laboratories in Japan, studying chirped DFB gratings. Since 1998, he has lectured in physics and optoelectronics at Kassel. From 2000 to 2002 , he was dean of the electrical engineering faculty. Hillmer is one of the founding members of the Center for Interdisciplinary Nanostructure Science and Technology (CINSaT) at Kassel.

Hillmer can be reached by e-mail at hillmer@uni-kassel.de.

Roland Germann, Guest Editor for this issue of MRS Bulletin, 


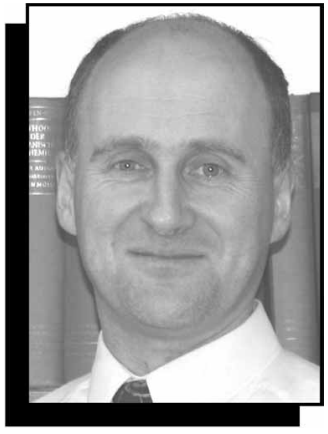

Hartmut Hillmer

joined the IBM Zurich Research Laboratory as a research staff member in 1990 following his graduation from the University of Stuttgart with a PhD degree in physics. His dissertation dealt with dry-etching of III-V semiconductors for the fabrication of nanostructures and distributed-feedback lasers as well as the optical and electrical characterization of dryetch-induced damage.

Germann's current work at IBM Zurich is focused on fabrication technology and materials issues for the development of optical planar lightwave circuits for components such as dynamic gain equalizers and dispersion compensators for networks based on wavelength-division multiplexing. Germann is a member of the German Physical Society.

$\mathrm{He}$ can be reached by e-mail at ger@ zurich.ibm.com.

Thomas Fuhrmann is a research assistant at the University of Kassel in Germany. His research involves the photonic applications of molecular glasses, particularly waveguide optics, photonic crystals, and self-organized nanostructures. He studied chemistry in Marburg, Germany, and Bologna, Italy, and did his PhD

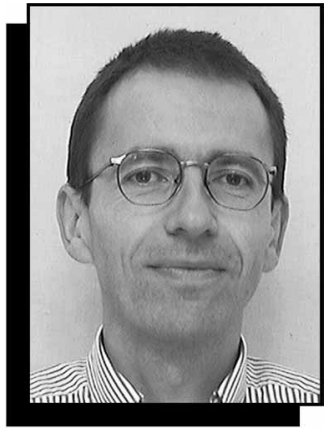

Roland Germann

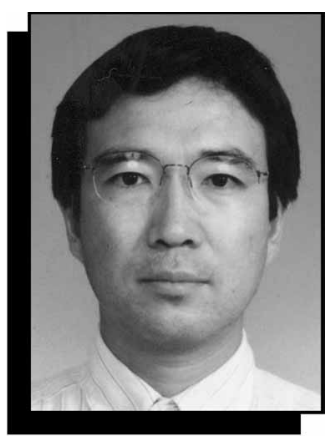

Yoshinori Hibino

work at Marburg University on functional polymers for optical data storage. After a two-year postdoctoral appointment at Kyushu University, Japan, where he worked in the field of organic light-emitting diodes, he joined

Duisburg University in 1999 as a member of the scientific staff and moved to the University of Kassel in 2001 to complete his habilitation.

Fuhrmann can be reached by e-mail at th.fuhrmann@ uni-kassel.de.

\section{Philippe Guenot is} in charge of a research group modeling the optical properties of silica fibers and their connection to glass structure at Alcatel Cable France.

He received an engineering degree in physics as well as a master's degree in microelectronics in 1991. In 1993, he joined the Alcatel Research Center as a PhD student, working on op-

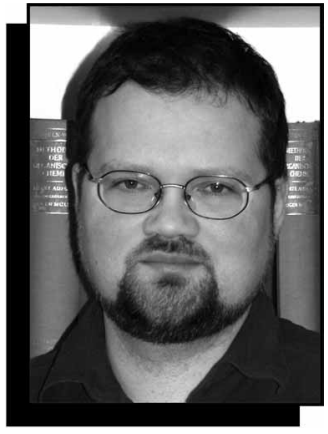

Thomas Fuhrmann

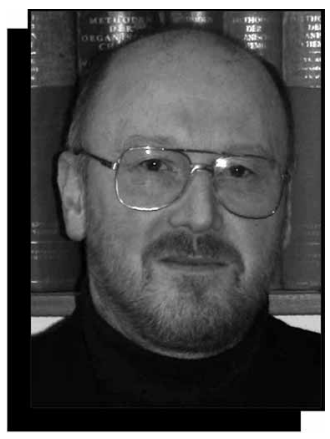

Josef Salbeck

tical properties of silica glass. He was awarded his $\mathrm{PhD}$ degree in 1997 from Paris-Sud Orsay University. He then joined Alcatel Cable as a research engineer in charge of optical loss modeling in transmission fibers before taking on his current position as group leader in 2000.

Guenot can be reached at Alcatel Cable France, 53 rue de Jean Broutin, F-78700 ConflansSainte-Honorine, France; and by e-mail at philippe.guenot@ alcatel.fr.

Andreas Hangleiter is a professor of physics at the Technical University of Braunschweig,

Germany, and director of the Institute of Technical Physics there. His early research focused on the electronic and optical properties of silicon. Later on, InP-based near-IR materials and optoelectronic device physics became the main focus of his work,

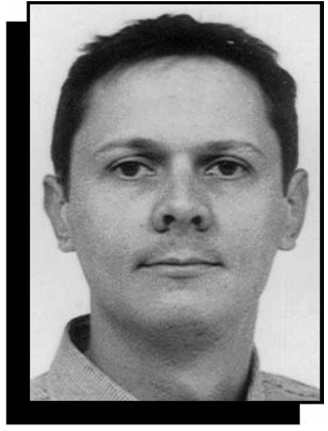

Philippe Guenot

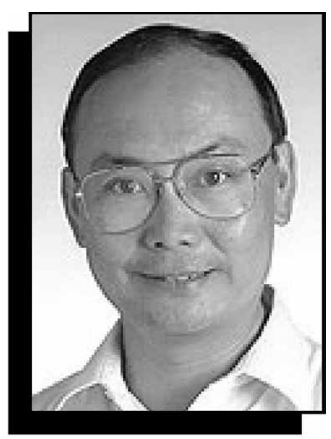

Charles W. Tu

with AlGaInP-based visible materials and nanostructures following in the mid-1990s. Since 1994, his work on III-nitrides has been at the center of his interest. Hangleiter received his diploma degree in physics in 1982 and his $\mathrm{PhD}$ degree in physics in 1985, both from the University of Stuttgart, Germany. From 1986 to 1987, he was a visiting researcher at IBM Research in Yorktown Heights, N.Y., returning to the University of Stuttgart as an assistant professor. From 1994 to 1998 , he was a Heisenberg fellow of the Deutsche Forschungsgemeinschaft.

Hangleiter can be reached by e-mail at a.hangleiter@tu-bs.de.

Yoshinori Hibino is a research scientist in the NTT Photonics Laboratories division of NTT Corporation in Kanagawa, Japan. His current research inter-

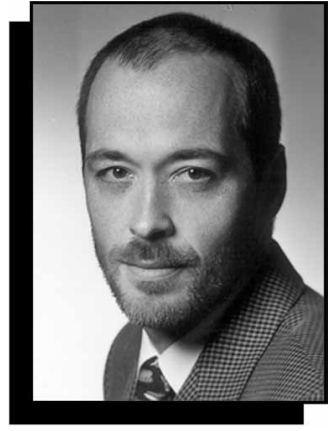

Andreas Hangleiter

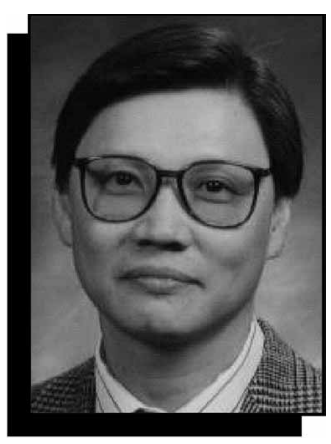

Paul K.L. Yu

ests are focused on planar lightwave circuits for photonics networks, such as arrayed waveguide gratings and optical switches. He received $\mathrm{BS}, \mathrm{MS}$, and $\mathrm{PhD}$ degrees in applied physics from the University of Tokyo, in 1980, 1982, and 1986, respectively. In 1982, he joined the Ibaraki Electrical Communication Laboratories of NTT Corp., where he was engaged in the research and development of optical fibers and planar lightwave circuits for telecommunications. He has published more than 100 technical articles in these research fields. From 1989 to 1990, he was a visiting research scholar at the Optical Sciences Center of the University of Arizona. Hibino is a member of the Institute of Electronics, Information, and Communication Engineers, and the Japanese Society of Applied Physics. 
Hibino can be reached by e-mail at yhibino@ aecl.ntt.co.jp.

Nada O'Brien is a product development manager at Optical Coating Lab Inc. (OCLI). She joined OCLI in 1993 after earning a BS degree in chemical engineering from Tri-State University, Indiana, and MS and PhD degrees in materials science and engineering from the University of Wisconsin-Milwaukee. She has over 10 publications in the field of thin-film coatings and processes.

O'Brien can be reached by e-mail at nada_obrien@ocli.com.

Josef Salbeck is a professor of macromolecular chemistry and molecular materials in the Physics Department at the University of Kassel, Germany. His research interests mainly involve functional molecular and hybrid materials and organic glasses for electronic, optical, and optoelectronic applications. After training and working as a chemical laboratory assistant, he studied chemistry at the University of Regensburg, graduating in 1985 from the Department of Chemistry and obtaining his PhD degree there in 1989. He finished his habilitation in organic chemistry in 1992 in the field of electrochemical-optical combination methods and held a lectureship in organic chemistry at the University of Regensburg. In 1993, Salbeck joined Hoechst AG in Frankfurt as laboratory leader of a research group working on light-emitting polymers within the Corporate Research division. As a Heisenberg fellow of the Deutsche

Forschungsgemeinschaft, he went to the Max Planck Institute for Polymer Research in Mainz in 1995 and was appointed a professor of electrochemistry and optoelectronic materials at Duisburg University in 1998. Since 2000, he has been a full professor at the University of Kassel and is one of the founders of the recently established Center for
Interdisciplinary Nanostructured Science and Technology there.

Salbeck can be reached by e-mail at salbeck@uni-kassel.de.

Robert Sargent is a director of process development at OCLI, a JDS Uniphase Company. He joined OCLI in 1989 after earning his BS degree in physics from the University of California, Berkeley, and MS and $\mathrm{PhD}$ degrees in Optical Sciences from the University of Arizona. Sargent's interests include thin-film process development and applications of thin films in optical systems.

Sargent can be reached by email at rsargent@ocli.com.

Charles W. Tu is a professor and chair of the Department of Electrical and Computer Engineering at the University of California, San Diego. His research centers on novel compound semiconductors grown by gas-source molecularbeam epitaxy for electronic and optoelectronic applications.
His group has also worked on selected-area regrowth by chemicalbeam epitaxy. Currently, his main focus is on low-bandgap dilute nitrides and their applications. He received a BSc degree in physics at McGill University in 1971, an MPhil degree in physics at Yale University in 1972, and a PhD degree in engineering and applied science at Yale in 1978. He was a lecturer in the Yale Physics Department from 1978 to 1980. From 1980 to 1988 , he was a member of the technical staff (MTS) at AT\&T Bell Laboratories in Murray Hill, N.J., where in 1987 he was named Distinguished MTS. In 1988, he joined the faculty of UCSD. He was appointed department chair in 1999. He is also an adjunct professor of the Department of Materials Science and Engineering at Kwang-ju Institute of Science and Technology in Korea. Tu has co-authored more than 290 journal papers and several book chapters. He is a fellow of the IEEE and the Ameri- can Physical Society and a long-time member of AVS, TMS, and MRS.

Tu can be reached by e-mail at ctu@ ece.ucsd.edu.

Paul K.L. Yu is a professor and vice chair of the Department of Electrical and Computer Engineering at the University of California, San Diego, where he has been on the faculty since 1983. At UCSD, he conducts research in materials and devices for fiber-optic and optoelectronics applications, in particular, for microwave transmission over fiber. His group has developed high-power optical detectors for microwave frequency detection, high-linearity waveguide modulators, and high-power semiconductor switches for microwave generation. $\mathrm{Yu}$ received a $\mathrm{PhD}$ degree in applied physics at the California Institute of Technology in 1983. He is a senior member of IEEE and a member of OSA.

Yu can be reached by e-mail at yu@ ece.ucsd.edu.

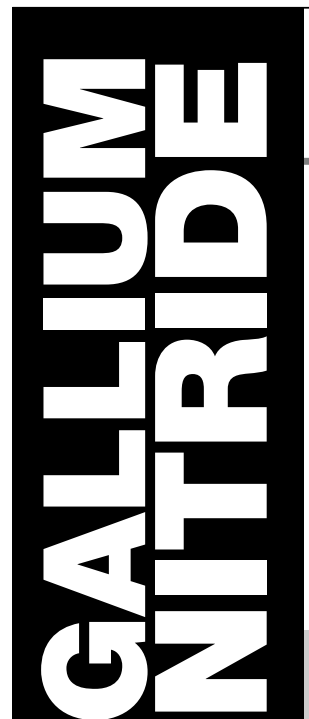

\section{Don't Miss These Publications on GaN and Related Alloys from the Materials Research Society}

GaN and Related Alloys-2002

Volume 743-B

\$104.00 MRS Member

$\$ 119.00$ U.S. List

$\$ 137.00$ Non-U.S.

GaN and Related Alloys-2001

Volume 693-B

$\$ 81.00$ MRS Member

$\$ 93.00$ U.S. List

$\$ 106.00$ Non-U.S.
GaN and Related Alloys-2000

Volume 639-B

$\$ 95.00$ MRS Member

$\$ 110.00$ U.S. List

$\$ 126.00$ Non-U.S.

GaN and Related Alloys-1999

Volume 595-B

$\$ 86.00$ MRS Member

$\$ 99.00$ U.S. List

$\$ 114.00$ Non-U.S.

NOTE: FREE online Web access for MRS members on all volumes listed above.

For more information, or to order any of the proceedings volumes listed above, contact the MRS Customer Services Department. 